\title{
Chinese National Condition Based Power Dispatching Optimization in Microgrids
}

\author{
Gang Chen, ${ }^{1}$ Jian-Ming Wang, ${ }^{2}$ Xiao-Dong Yuan, ${ }^{2}$ Liang Chen, ${ }^{2}$ \\ Lu-Jia Zhao, ${ }^{3}$ and Yu-Ling He $\mathbb{1}^{3}$ \\ ${ }^{1}$ State Grid Jiangsu Electric Power Company, Nanjing, Jiangsu, China \\ ${ }^{2}$ State Grid Jiangsu Electric Power Company Research Institute, Nanjing, Jiangsu, China \\ ${ }^{3}$ Faculty of North China Electric Power University, Baoding, Hebei, China \\ Correspondence should be addressed to Yu-Ling He; heyuling1@163.com
}

Received 4 August 2017; Revised 10 January 2018; Accepted 22 January 2018; Published 14 March 2018

Academic Editor: Daniela Proto

Copyright (C) 2018 Gang Chen et al. This is an open access article distributed under the Creative Commons Attribution License, which permits unrestricted use, distribution, and reproduction in any medium, provided the original work is properly cited.

\begin{abstract}
This paper proposed a study on the power dispatching optimization in the microgrid aiming at Chinese national condition based on PSO algorithm. The whole work is on the basis of the weighted factor variation of the objective function due to different weather conditions. Three cases including the good contamination-diffusing weather condition, the smog weather condition, and the normal condition are considered, respectively. In the case of smog weather, the new energy generation and the battery system will be all out to use as less power as possible from the primary grid so that the pollution produced by coal consumption in the thermal power plants can be upmost reduced. However, in the case of perfect contamination-diffusing weather, the battery is not used to reserve its lifetime, while a large amount of exchanged power from the primary grid is used to obtain a most economic-efficient effect. In normal condition, the power dispatching is performed in a most balanced way considering not only the cost but also the environmental management. The case study in Suzhou Industrial Part confirms the effectiveness of the proposed method in this paper.
\end{abstract}

\section{Introduction}

As the rapid economic development, the electric power requirement is increasing. However, the primary energy source has become a serious problem as well as the environmental pollution $[1,2]$. It is significant to develop the sustainable energy based power generation technologies such as wind generation and photovoltaic (PV) generation [3].

Typically, as the wind generation and the photovoltaic generation are connected to the power grid with a larger and larger capacity, the voltage and frequency stability of the grid has become a serious problem. To solve this problem, the active distribution network (ADN) [4] and the optimal allocation of dispersed energy storage systems in AND [5] are employed. Hereafter, many effective methods, such as the generalized model of VSC-based energy storage systems [6] and the deployment strategy taking the two-stage solar photovoltaic-based stand-alone scheme and having battery as energy storage element [7], are proposed to secure the transient stability and the stationary stability. Moreover, the construction of smart grid which contains the microgrids and the distributed network is being carried out powerfully all over the world $[8,9]$, and the demand response management [10], the maximum power point tracking of the photovoltaic systems for fast changing environmental conditions [11], the power loss [12], and especially the scientific power dispatch [13] inside the microgrids have been great challenges.

By far, both the AC microgrids and the DC microgrids have found the significance of optimization-based energy management (EM) [14-17]. It is shown that the bilevel control scheme (BLCS) with primary and secondary controllers is an effective method in achieving optimal dispatch of generation resources in systems with multiple microgrids [18]. Since microgrid's economic load dispatch normally does not support distributed networks, especially when they belong to different companies and share different interests, it is significant to consider a dynamic electricity pricing policy to 
achieve optimal operation of distributed network with multimicrogrids [19]. This kind of policy now has been carried out in many countries as well as China. Then the further problem is how to dispatch the power output of the new energy in the microgrid as well as the exchanged power from the main grid in different time periods to not only satisfy the consumption request but also get a minimum cost.

Usually, the power dispatch is considered within 24 hours as a typical cycle which can be classified into critical peak demand period, normal demand period, and valley demand period, of which the electricity prices are pretty different [20]. Nikmehr et al. have found that PSO algorithm is qualified to minimize the cost function and regulate the power demand and power transaction between each microgrid and the main grid [21], while Liao employed the chaotic quantum genetic algorithm to solve the environmental economic dispatch problem [22]. Koller et al. defined a degradation cost function to optimally control the battery energy storage system [23], and Su et al. discussed how to scientifically use the renewable sources in the microgrids for an optimal energy scheduling [24].

However, most of the researches set up only one objective function aiming at getting the minimum cost as well as the balance among the consumption demand, the economicefficiency, and the environmental management, while they rarely considered the impact of the special regional condition such as the local policy and the weather condition for contamination-diffusing on the power dispatching scheme. Unfortunately, the reality may request more than one optimization scheme due to different running conditions [25]. This multicondition requested situation now is very common in China, especially in the North area in winter $[26,27]$. For example, when there is smog weather during the winter, the new energy based power generation is requested to be fully used while thermal power plant is expected to restrain its output to protect the environment, regardless of the cost under the guidance of the national and local policies [28, 29]. On the contrary, when there is perfect contaminationdiffusing weather, it is permitted that the microgrid consumes more energy from the main grid produced by thermal power plants [30], which may lead to a restrain to the new energy generation due to its higher cost.

As an improvement, this paper investigates the power dispatching optimization in microgrids aiming at Chinese national conditions based on PSO algorithm. The work takes the Suzhou Industrial Part as the study object and generally considers three conditions, that is, the good contaminationdiffusing weather condition, the smog weather condition, and the normal condition. The construction of the whole paper is as follows. In Section 2, the PSO algorithm for a special use under the Chinese national condition for the power dispatching optimization will be proposed. In Section 3, PSObased power dispatching realizations for three performing conditions in Suzhou Industrial Part will be carried out, respectively. Comparison and discussion will be taken as well in this section to obtain a useful qualitative result. Finally, the main conclusions will be drawn in Section 4.

\section{Optimization Scheme Based on PSO Algorithm}

2.1. Boundary Condition and Limitation. For the battery, neither overcharge nor overdischarge is permitted. Instead, the state-of-charge (SOS) of the battery should meet

$$
S_{\min } \leq S_{t} \leq S_{\max }
$$

where $S_{t}$ is the SOC at period $t$, while $S_{\min }$ and $S_{\max }$ are the minimum value and the maximum value of SOC, respectively. Usually, $S_{\min }$ is set to 0.3 while $S_{\max }$ is set to 0.95 .

During the unit period $\Delta t$, the charge and discharge power of the battery should be balanced, and the value of SOC is defined as

$$
S_{t}=S_{0}+\frac{\sum_{t=1}^{T} P_{\text {cha }, t} X_{t} \Delta t-\sum_{t=1}^{T} P_{\mathrm{dis}, t} Y_{t} \Delta t}{E_{b}}
$$

$$
X_{t} \cdot Y_{t}=0 \text {, }
$$

where $S_{0}$ is the initial state of SOC, $P_{\text {cha, } t}$ and $P_{\text {dis }, t}$ are the charge power and the discharge power at period $t$, respectively, $X_{t}$ and $Y_{t}$ stand for the charge and discharge status, respectively, $X_{t} \in\{0,1\}, Y_{t} \in\{0,1\}$, and $T$ is the total period number.

Meanwhile, considering that the charge-discharge power and the charge-discharge frequency are sensitively related to the battery lifetime, the maximum charge and discharge power each time is set no more than $20 \%$ of the rated capacity $E_{b}$, while the charge-discharge frequency is restrained by the upper thresholds indicated in

$$
\begin{aligned}
0 & \leq P_{\text {cha }, t} \leq 0.2 E_{b} X_{t} \\
0 & \leq P_{\text {dis }, t} \leq 0.2 E_{b} Y_{t} \\
\sum_{t=1}^{T}\left|X_{t+1}-X_{t}\right| & \leq N_{1} \\
\sum_{t=1}^{T}\left|Y_{t+1}-Y_{t}\right| & \leq N_{2} .
\end{aligned}
$$

Besides the previously mentioned constrain conditions, the load and the power should still meet

$$
P_{\text {load }}=P_{\text {exc }}+P_{\text {pho }}+P_{\text {win }}+P_{\text {bat }} \quad P_{\text {exc }} \leq P_{\text {lim }},
$$

where $P_{\text {exc }}$ is the exchanging power between the micro and the primary grid, $P_{\text {load }}$ is the consuming power of the load in the microgrid, $P_{\text {pho }}$ is the photovoltaic generation power, $P_{\text {win }}$ is the wind generation power, and $P_{\text {bat }}$ is the power provided by the battery, and $P_{\text {lim }}$ is the utmost power that the microgrid can obtain from the primary grid.

\subsection{Special PSO Algorithm for Chinese National Condition}

2.2.1. PSO Algorithm Application. Particle Swarm Optimization (PSO) simulates the birds' food seeking behavior. Each bird is a particle [31]. For the power dispatching in the microgrid, each power output scheme, including the wind generation output, the PV generation output, the storage output, and the exchanged power from the main grid, is a particle. The particle swarm is a matrix, while the particle structure is actually an array vector which can be indicated as

$$
P_{i}=\left[P_{\text {win }}(i), P_{\mathrm{ph}}(i), P_{\mathrm{bat}}(i), P_{\mathrm{exc}}(i)\right] \text {. }
$$


TABLE 1: Weather condition mode criterion.

\begin{tabular}{|c|c|c|c|c|c|c|}
\hline AQI and wind data & $\begin{array}{c}\mathrm{AQI}<=50 \text { \& with } \\
\text { wind }\end{array}$ & $\begin{array}{c}\mathrm{AQI}<=100 \& \\
\text { wind speed }>3 \mathrm{~m} / \mathrm{s}\end{array}$ & $\begin{array}{c}100<\mathrm{AQI}<150 \& \\
\text { with wind }\end{array}$ & $\begin{array}{c}150<\mathrm{AQI}<=180 \\
\& \text { wind speed }> \\
3 \mathrm{~m} / \mathrm{s}\end{array}$ & $\begin{array}{l}180<\mathrm{AQI}<250 \& \\
\text { wind speed }<3 \mathrm{~m} / \mathrm{s}\end{array}$ & $\mathrm{AQI}>=250$ \\
\hline Weather condition & $\begin{array}{l}\text { Good contamir } \\
\text { weather }\end{array}$ & $\begin{array}{l}\text { hation-diffusing } \\
\text { condition }\end{array}$ & \multicolumn{2}{|c|}{ Normal condition } & \multicolumn{2}{|c|}{ Smog weather condition } \\
\hline
\end{tabular}

Each particle has a fitness value for the objective function, as well as a speed to determine its forward direction and distance. This algorithm needs iterations to get the optimal power dispatching scheme. During the iteration each particle refreshes its own value according to the boundary condition and limitation described in Section 2.1. For each particle, it has two extreme values, that is, the currently individual best solution value which is usually written as $p$ Best, and the currently general best solution value which is usually written as $g$ Best.

Generally, the special PSO algorithm for Chinese national condition follows the following steps.

(1) Condition Confirmation. Since there are three weather conditions relating to three different objective functions (see Section 2.2.2), the first performance is to check the weather condition mode according to the air quality index (AQI) and wind data from the weather bureau. The weather condition mode criterion taken in this paper is indicated in Table 1.

After the weather condition is confirmed, the corresponding objective function will be distinguished. Then the power supply combination scheme and the weighted factor for each kind of power output will varied. More details will be described in Section 2.2.2.

(2) Initialization. The initial position of Particle $i$ is

$$
x_{i d}^{0}=r_{3}\left(x_{d}^{M}-x_{d}^{m}\right)+x_{d}^{m}
$$

where $r_{3}$ is a random between 0 and 1 and $x_{d}^{M}$ and $x_{d}^{m}$ are the maximum and the minimum values of the $d$ th component of the location variation, respectively. In this paper $d=1,2,3,4$, respectively, denoting the four types of the power output, that is, the wind generation output, the PV generation output, the storage output, and the exchanged power from the main grid. And the initial velocity of Particle $i$ is

$$
v_{i d}^{0}=r_{4} v_{d}^{M}
$$

where $r_{4}$ is a random between 0 and 1 and $v_{d}^{M}$ is the max velocity of the $d$ th component of the particle. Usually, $v_{d}^{M}=$ $0.1 x_{d}^{M}$.

In PSO algorithm, the inertia weight factor $w$ is an important parameter that determines the general and the regional search ability. Yuhui Shi proposed the improved PSO in 1998 by optimizing $w$. It is shown that PSO can get a rapid convergence under a descending strategy when $w$ is in the range of $[0.4,0.9]$, while the algorithm may fall into a regional optimization when $w>1.2$. $w$ can be calculated via

$$
w=w_{\text {start }}-\frac{t\left(w_{\text {start }}-w_{\text {end }}\right)}{T}
$$

where $w_{\text {start }}=0.9, w_{\text {end }}=0.4, t$ is the current iterating value, and $T$ is the maximum value of the iteration.

Besides $w$, there are still another two parameters, that is, $c_{1}$ and $c_{2}$, which, respectively, determine the effects of the individual history and the influence of the general swarm's experience, on the particle motion trial. During the optimization calculation, usually $c_{1}=c_{2}=2$. In this paper, we use the selfadapted method proposed by Ratnaweera et al., to solve the over-early convergence problem for a better optimizing effect. $c_{1}$ and $c_{2}$ can be obtained by

$$
\begin{aligned}
& c_{1}=\frac{\left(c_{1 f}-c_{1 e}\right) t}{T}+c_{1 e} \\
& c_{2}=\frac{\left(c_{2 f}-c_{2 e}\right) t}{T}+c_{2 e},
\end{aligned}
$$

where $c_{1 e}$ and $c_{1 f}$ are the beginning and final value of $c_{1}$, respectively, while $c_{2 e}$ and $c_{2 f}$ are the beginning and final value of $c_{2}$, respectively. In this paper, we wet $c_{1 e}=2.5, c_{1 f}=$ $0.5, c_{2 e}=0.5$, and $c_{2 f}=2.5$

(3) Particle Fitness Judgment. If the current fitness value of the particle is better than its former value, the velocity and the location of the particle will be updated. Then, the general optimized values of the whole swarm will be searched and updated as the latest velocity and location.

(4) Iteration Monitoring. The iteration will not stop and turn to step (3) for the further calculation until the interval number reaches the setting threshold or the result obtains a better accuracy than the setting one.

2.2.2. Objective Functions and Optimization Scheme. The general idea for the power dispatching optimization is as follows.

Typically, due to different running conditions, the objective function varies among the three cases. Case 1: there is no battery and no power exchange limit between the microgrid and the primary grid, while the minimum average unit power cost is set as the objective function. Case 2: the battery is taken into action, and the power exchange between the microgrid and the primary grid is $45 \mathrm{MW}$. Meanwhile, the wind generation and the photovoltaic generation are fully used. The objective function is still the minimum average unit power cost. Case 3: the battery is on work and the power exchange limit is also $45 \mathrm{MW}$, while the objective function is the minimum total generation cost.

Correspondingly, case 1 is for the perfect contaminationdiffusing weather condition, case 2 is for the smog weather 
condition, and case 3 is for the normal condition. The flowchart for normal condition is indicated in Figure 1, while the working process logics for the other two conditions are generally similar, with the differences in the storage system use and the exchanged power limitation between the microgrid and the primary grid.

$$
\min C_{L}= \begin{cases}w_{1 N} \cdot C_{\mathrm{pho}}+w_{2 N} \cdot C_{\mathrm{win}}+w_{3 N} \cdot C_{\mathrm{bat}}+w_{4 N} \cdot \sum_{t=1}^{T} R_{\mathrm{exc}} P_{\mathrm{exc}}(t)+w_{5 N} \cdot C_{\mathrm{env}} & \text { (normal) } \\ w_{4 S} \cdot \sum_{t=1}^{T} R(t) P_{\mathrm{exc}}(t)+w_{5 S} \cdot C_{\mathrm{env}} & \text { (smog weather) } \\ w_{1 G} \cdot C_{\mathrm{pho}}+w_{2 G} \cdot C_{\mathrm{win}}+w_{3 G} \cdot C_{\mathrm{bat}}+w_{4 G} \cdot \sum_{t=1}^{T} R(t) P_{\mathrm{exc}}(t)+w_{5 G} \cdot C_{\mathrm{env}} & \text { (good weather) }\end{cases}
$$

where

$$
\begin{aligned}
C_{\text {pho }} & =\sum_{t=1}^{T} R_{\text {pho }}(t) P_{\text {pho }}(t) \\
C_{\text {win }} & =\sum_{t=1}^{T} R_{\text {win }}(t) P_{\text {win }}(t) \\
C_{\text {bat }} & =\sum_{t=1}^{T} R_{\text {bat }}(t) P_{\text {bat }}(t) \\
C_{\text {env }} & =\sum_{t=1}^{T} R_{\text {env }}(t) P_{\text {exc }}(t) \\
T & =24 \times \frac{60}{n_{T}} .
\end{aligned}
$$

And $w_{1 N}$ to $w_{5 N}$ are the weighted factors for normal condition, $w_{4 S}$ and $w_{5 S}$ are the weighted factors for smog weather, and $w_{1 G}$ to $w_{5 G}$ are the weighted factors for good contamination-diffusion weather. In different cases, the weighted factors are assigned with different values. For example, $w_{5 G}$ is smaller than $w_{5 N}$ and much smaller than $w_{5 S} . C_{\text {pho }}, C_{\text {win }}, C_{\text {bat }}$, and $C_{\text {env }}$ are the costs of the photovoltaic generation, the wind generation, the battery charge and discharge, and the environmental protection, respectively, $R(t)$ and $P_{\text {exc }}(t)$ are the unit price to buy the power from the primary and the power the microgrid absorbs from the primary grid at period $t$. $R_{\text {pho }}(t)$ and $P_{\text {pho }}(t), R_{\text {win }}(t)$ and $P_{\text {win }}(t), R_{\text {bat }}(t)$ and $P_{\text {bat }}(t)$, and $R_{\text {env }}(t)$ and $P_{\text {env }}(t)$ are the unit prices and consumed power at period $t$ for the photovoltaic generation, the wind generation, the battery charge-discharge, the environmental governmental cost, respectively. $T$ is the total number of the periods, while $n_{T}$ is the lasting time that each period contains.

To obtain the accurate value of each weighted factor, we employ the Analytic Hierarchy Process (AHP) method to get the weighted factors for the three conditions. For normal condition, the five weighted factors are treated generally equal, while in the haze weather condition the environmental management cost factor is treated as the most primary one which is followed with the power exchange cost between the microgrid and the primary grid. In this case the cost of the new energy generation and battery system is ignored due to the government policy. However, in the case of perfect contamination-diffusion weather, the priority orders are $w_{3 G}$, $w_{4 G}, w_{2 G}, w_{1 G}$, and $w_{5 G}$.

To better dispatch the power inside the microgrid, the 24 hours are divided into 96 periods in general and each period is 15 minutes. These 96 periods are further classified into three sections, namely, the peak section, the valley section, and the stable section. According to the historical power consuming data, the load and the generation output in the microgrid are predicted, and the battery SOC is monitored.

During the peak sections, when the wind generation and the photovoltaic generation are sufficient, the tactic is set to sell the power generated by the microgrid as much as possible. If the sold power is less than the maximum capacity $P \max$ of the new energy, make the battery discharge preferentially and then dispatch the power generated by the wind and solar. If the wind and photovoltaic generation power is still redundant, use the surplus power to charge the battery.

During the valley and stable sections, if the wind and the photovoltaic generation power is sufficient, charge the battery in advance and then sell the power to the primary grid.

During the valley sections, if the wind and photovoltaic generation power is not sufficient, buy power from the primary grid with priority, and dispatch the power generated by the wind and solar energy if the bought power is more than $P$ max. Meanwhile, charge the battery.

During the stable sections, if the wind and photovoltaic generation is not sufficient, dispatch the new energy power firstly and then buy power from the primary grid when the consumed new energy power is more than Pmax. Meanwhile, the battery is under the charge state as well.

2.2.3. Method to Get Weighted Factors. There are generally three steps to obtain the weighted factors

(1) Set up the Layer Structure and the Judge Matrix. The layer structure is set up as Figure 2, where the voltage stability of the 3rd layer is ensured by the exchanged power limitation. Generally, the 5 factors in the 3rd layer are the essential ones, whose judge matrix can be indicated as

$$
A^{*}=\left[\begin{array}{cccc}
a_{11} & a_{12} & \cdots & a_{15} \\
a_{21} & a_{22} & \cdots & a_{25} \\
\cdots & \cdots & \cdots & \cdots \\
a_{51} & a_{22} & \cdots & a_{55}
\end{array}\right],
$$




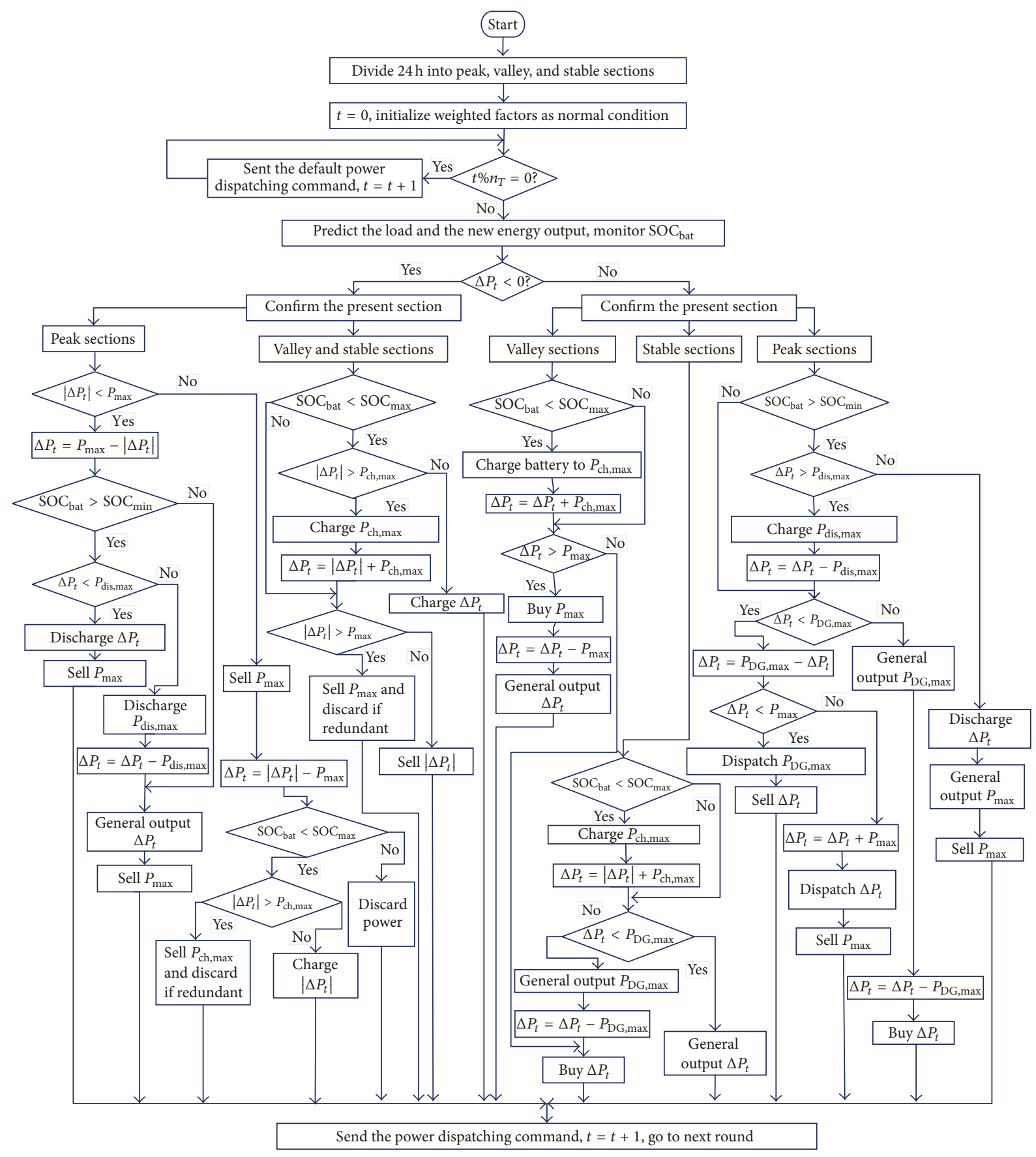

FIGURE 1: The general flow chart of the scheme for normal condition.

where $a_{i j}$ is obtained by comparing the importance of factor $i$ with factor $j$ in the same layer. The first row to the last row and the first volume to the last volume, respectively, stand for the relative importance among the wind generation, the photovoltaic generation, the battery system, the power exchange between the microgrid and the primary grid, and the environmental management. For example, $a_{11}$ to $a_{1 n}$ denote the relative importance for the wind generation comparing with the photovoltaic generation, the battery system, the power exchange, and the environmental management. They can be indicated by the indexes of 1 to 9 and their reciprocals, with the meanings in Table 2.

(2) Calculated the Weighted Factors. Firstly compute the element product of each row.

$$
M_{i}=\prod_{j=1}^{5} a_{i j}, \quad i=1,2, \ldots, 5 .
$$


TABLE 2: Forming rules of the judge matrix.

\begin{tabular}{lc}
\hline Index & Meaning \\
\hline 1 & Equally important \\
3 & A bit more important \\
5 & Evidently important \\
7 & Intensively important \\
9 & Extremely important \\
$2,4,6,8$ & Between the previous two adjacent indexes \\
reciprocals & Judge $a_{i j}$ when factor $i$ compares with $j$, while judge $a_{j i}=1 / a_{i j}$ when factor $j$ compares with $i$ \\
\hline
\end{tabular}

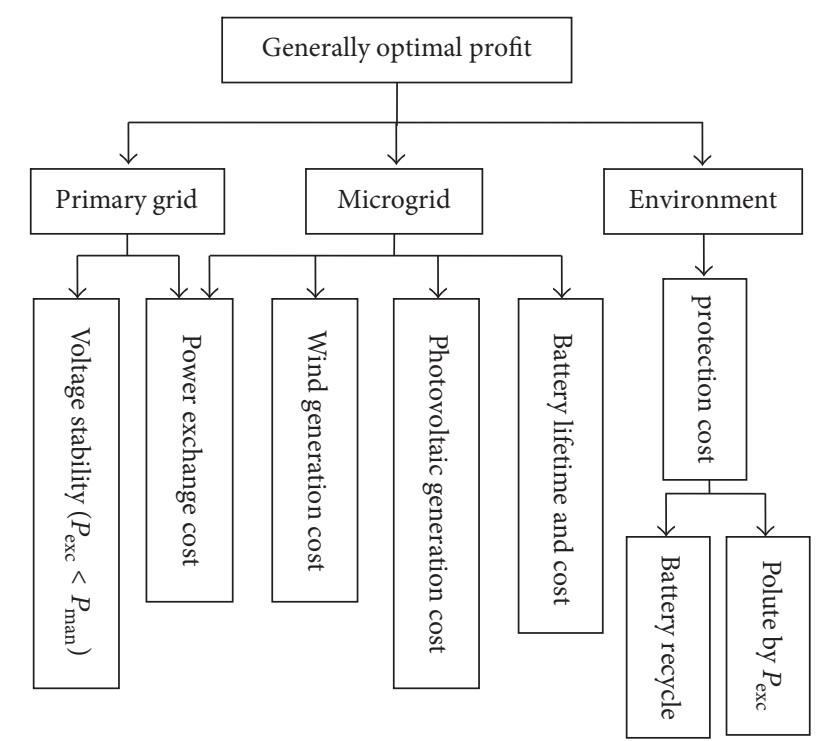

FIGURE 2: Layer structure diagram.

Secondly calculate the fifth root of $M_{i}$.

$$
k_{i}=\sqrt[5]{M_{i}}, \quad i=1,2, \ldots, 5
$$

Thirdly normalize the vector $K=\left[k_{1}, k_{2}, k_{3}, k_{4}, k_{5}\right]^{T}$ via

$$
w_{i}=\frac{k_{i}}{\sum_{i=1}^{5} k_{i}}, \quad i=1,2, \ldots, 5 .
$$

Finally form the weighted factor vector as

$$
W=\left[w_{1}, w_{2}, w_{3}, w_{4}, w_{5}\right] .
$$

(3) Consistency Verification. The consistency verification is carried out by bringing in an extra index CR which can be written as

$$
\begin{aligned}
\mathrm{CR} & =\frac{\mathrm{CI}}{\mathrm{RI}} \\
\mathrm{CI} & =\frac{\lambda_{\text {max }}-n}{n-1} \\
\lambda_{\text {max }} & =n,
\end{aligned}
$$

where RI can be found in Table 3.
If $\mathrm{CR}$ is equal to or smaller than 0.01 , it is thought that the consistency is qualified; otherwise the weighted factors should be recomputed. Usually, to make the consistency better, the domain is set less than 7. In this paper the domain $n$ is 5 .

\section{Power Dispatching Optimization Case Study}

3.1. General Introduction to Study Object. Suzhou Industrial Part, which is set up in 1994, is a key cooperative project between China and Singapore, covering 278 square kilometers. Inside the park there are more than 800 financial institutions, more than 30 public technology service platforms and 20 national innovation bases, 28 colleges of China and foreign countries, 480 research-development institutions, and more than 10 national scientific institutes. There are also $156 \mathrm{key}$ projects carried out by 92 of the world top 500 enterprises and 92 headquarters of the city-level organizations.

Currently, the park includes an international scientific section, a creative industry section, a Suzhou nanometer section, and a biological industry section which consumes a large amount of electric power. It is shown that Suzhou is one of the cities which consume the most electric power in China.

The microgrid of Suzhou Industrial Part consists of different types of loads including 2 charging stations for more than 1000 electric vehicles and 100 distributed generations including $25 \mathrm{MW}$ photovoltaic generation, $50 \mathrm{MW}$ wind generation, and $22 \mathrm{MW}$ storage capacity, as indicated in Figure 3. The power output of the wind generation and the photovoltaic generation is indicated in Figure 4, where the typical load distribution in the whole day is illustrated as well. To better demonstrate the amount comparison between the generation output and the consumption power, in Figure 4 the photovoltaic generation output is superimposed to the wind generation output.

It is suggested from Figure 4 that the new energy generation output fluctuates seriously. Therefore, the scientific power dispatching inside the microgrid is significant. The power price for selling and purchasing is indicated in Table 4, while the unit power cost is shown in Table 5.

3.2. Case 1 Study. Under the perfect contamination-diffusing weather condition, the battery is not used in order to reserve its lifetime. In this case, there is no particular restrict to the exchanging power between the microgrid and the primary 
TABLE 3: Value of RI for different domains.

\begin{tabular}{lllccccccccc}
\hline Domain $n$ & 1 & 2 & 3 & 4 & 5 & 6 & 7 & 8 & 9 & 10 & 11 \\
\hline RI & 0 & 0 & 0.58 & 0.9 & 1.12 & 1.24 & 1.32 & 1.41 & 1.45 & 1.49 & 1.51 \\
\hline
\end{tabular}

TABLE 4: Power prices in different times of the whole day (unit: RMB/kWh).

\begin{tabular}{lcccccc}
\hline Times & $0: 00-7: 00$ & $7: 00-10: 00$ & $10: 00-15: 00$ & $15: 00-18: 00$ & $18: 00-21: 00$ & $21: 00-24: 00$ \\
\hline Price to sell power & 0.22 & 0.42 & 0.65 & 0.42 & 0.65 & 0.42 \\
Price to buy power & 0.25 & 0.53 & 0.82 & 0.53 & 0.82 & 0.53 \\
\hline
\end{tabular}

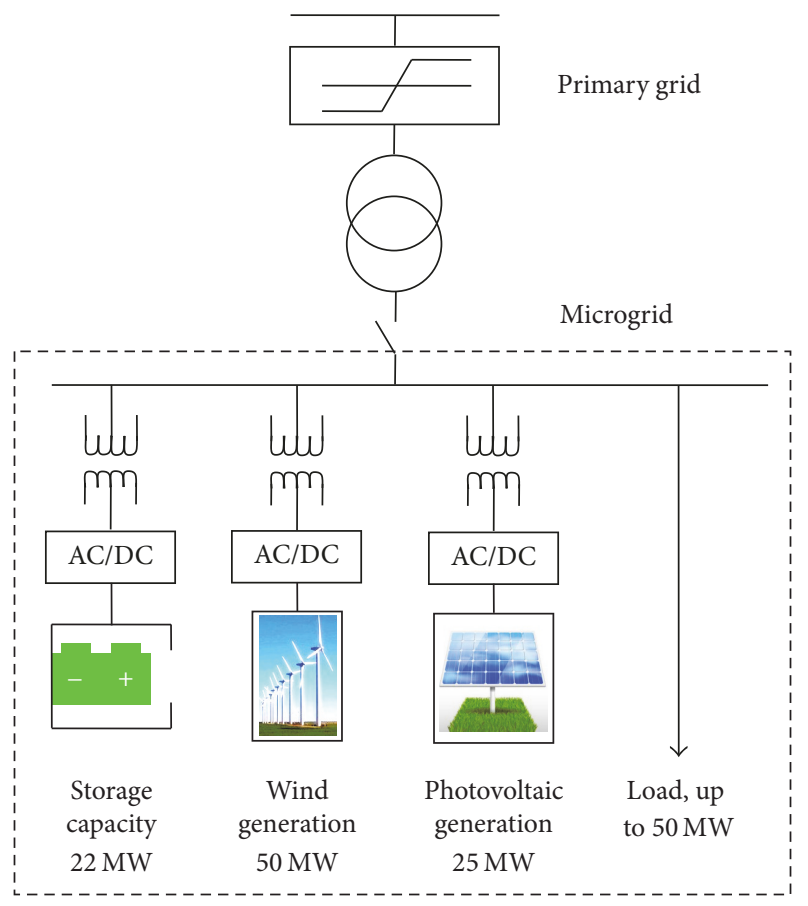

Figure 3: Constitution of microgrid of Suzhou Industrial Park.

grid. And the wind generation power, the photovoltaic generation power, and the exchanged power from the primary grid are indicated in Figure 5.

In this case, the power consumption for a whole day costs $316724 \mathrm{RMB}$, with the unit power cost at $0.33 \mathrm{RMB} / \mathrm{kWh}$. Due to the varying price both for power buying and selling, the wind generation output and the photovoltaic generation output are changing during different time periods. It is suggested from Figure 5 that the wind generation has a higher use rate than the photovoltaic generation. During 0:00 to 10:00 the wind generation and the photovoltaic generation have almost no power output, while during 10:00 to 23:00 the wind generation keeps exporting power all the time. The photovoltaic generation generally only works during 12:00 to 14:00, in which period the wind generation has the equivalent power output as the photovoltaic generation. The power gap between the load and the new energy generation output will be fulfilled by the exchange power from the primary grid.

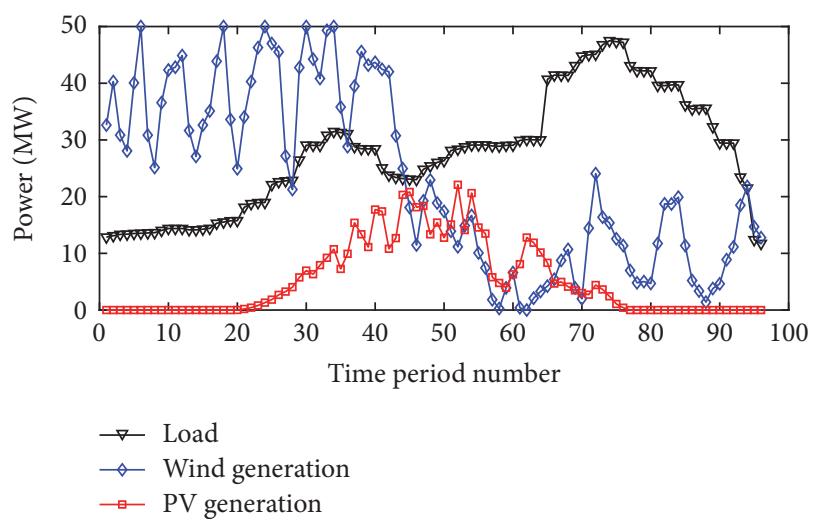

FIGURE 4: New energy power generation output and load distribution.

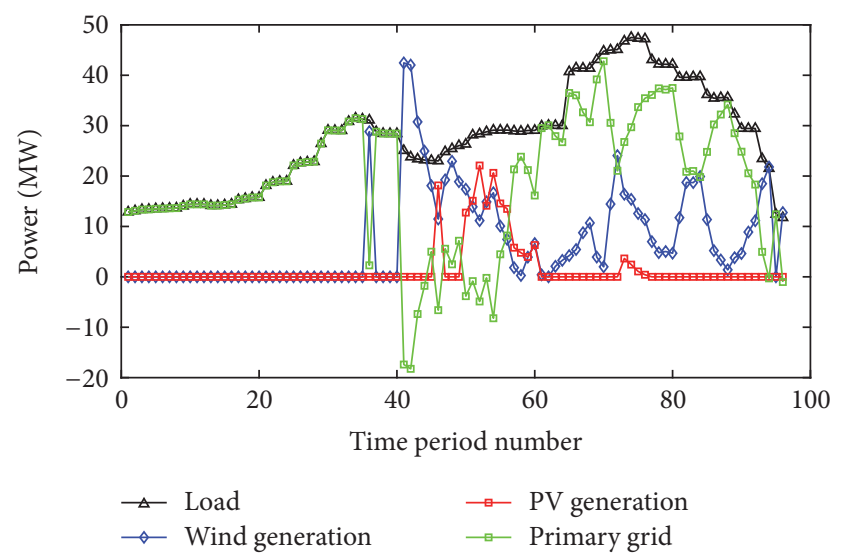

FIGURE 5: Power dispatching in good contamination-diffusing weather conditions.

3.3. Case 2 Study. Under the smog weather condition, the Chinese government has carried out specific policies to improve the air condition, including constraining the thermal power generation due to its smog emission. In this case, there is a restrict requiring less than $45 \mathrm{Mw}$ for the power exchanging between the microgrid and the primary grid. Meanwhile, the new energy generation and the battery are required an utmost use while the cost is put into the secondary position. The power dispatching for each kind of generation is indicated in Figure 6. 
TABLE 5: Power cost for different generation type.

\begin{tabular}{lccc}
\hline Type & Wind generation & Photovoltaic generation & Battery cost for once charge-discharge \\
\hline Cost & $0.52 \mathrm{RMB} / \mathrm{kWh}$ & $0.75 \mathrm{RMB} / \mathrm{kWh}$ & $0.2 \mathrm{RMB} / \mathrm{kWh}$ \\
\hline
\end{tabular}

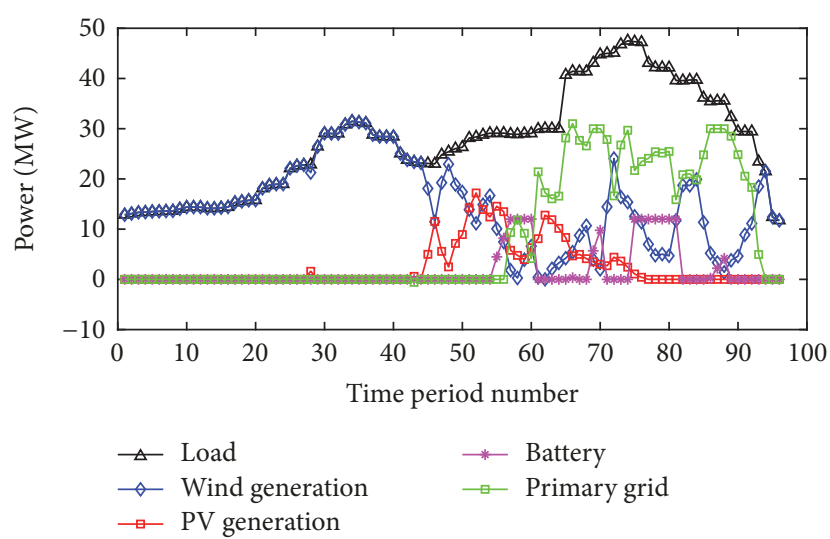

FIGURE 6: Power dispatching in smog weather condition.

The general cost for 24 hours in this case is $405670 \mathrm{RMB}$, and the unit power cost is $0.427 \mathrm{RMB} / \mathrm{kWh}$. It can be seen that the battery is employed in the two periods during 13:00 to $14: 00$ and 18:00 to $20: 00$ when the price for buying power from the primary grid is most expensive. This has somewhat obtained a good economic effect. However, since currently the power generation of the new energy is much inferior in cost to the traditional thermal power generation, the total cost for a whole day in this case is much higher than that in case 1.

3.4. Case 3 Study. In normal condition, the wind generation, the photovoltaic generation, and the battery are performed in a most balancing way considering not only the economic factors but also the environmental protection effects. The power dispatching for each kind of generation is indicated in Figure 7.

In this case, the general power cost for 24 hours is 358866.2 RMB, while the unit power cost is $0.378 \mathrm{RMB} / \mathrm{kWh}$. As indicated in Figure 7, the wind generation outputs power during the 40 th to the 60 th periods (10:00 to 15:00) and the 72 nd to 96 th periods (18:00-24:00), while the photovoltaic generation generally performs during the 40th to the 60th periods (10:00 to $15: 00)$ only. Similarly, the battery is employed mainly during the 46th to 60th periods (11:30$15: 00)$. It is easy to find that these periods generally cover the most expensive time sessions of power purchasing from the primary grid.

3.5. Comparison and Discussion. To obtain more details about the power dispatching of each kind of generation, comparison curves for the wind generation, the photovoltaic generation, the battery discharging, and the exchanged power from the primary grid, are indicated in Figures $8(\mathrm{a}), 8(\mathrm{~b})$, $8(\mathrm{c})$, and $8(\mathrm{~d})$, respectively.

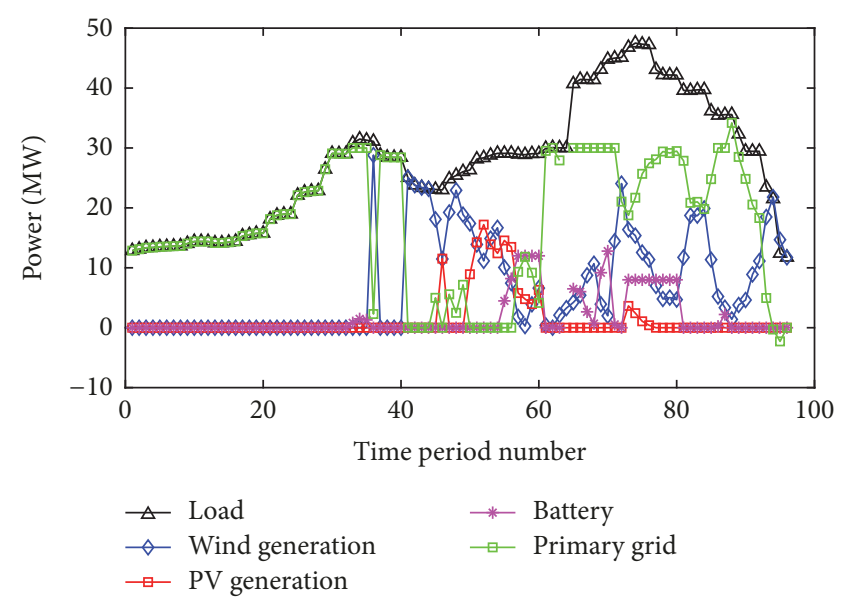

FIGURE 7: Power dispatching in normal condition.

As indicated in Figure 8(a), the wind generation power dispatching for different cases is greatly different during periods 1 to $40(0: 00-10: 00)$, while it is very similar for the three cases during periods 44 to 92 (11:00-23:00). Moreover, between 0:00-10:00, only in case 2 (smog weather case) the wind generation outputs the power and fulfills the load, while in other cases it is rarely used due to its higher cost than the traditional thermal power generation.

For the photovoltaic generation showing in Figure 8(b), it outputs more power during 11:00-15:00 in good contamination-diffusing weather conditions than other cases due to the good weather (usually also with a perfect sunshine) and the relatively low cost. However, in smog weather condition, the power output of the photovoltaic generation will have less fluctuation due to the environmental management request (it will try best to output as much power as possible), though constrained by the sunshine intensity the power output is less than case 1 . In normal condition, the photovoltaic generation outputs the minimum power among the three cases.

For the battery discharging, it is illustrated in Figure 8(c) that the battery outputs more power than in normal condition, particularly between 18:00-20:00 (periods 72-80), while in the good contamination-diffusing weather condition the battery is not used so that its lifetime can be stored for the future use.

When it comes to the exchanged power from the primary grid, as indicated in Figure 8(d), it confirms the dominant position of the thermal power generation in China (the power from the primary grid generally comes from the thermal power plants). No matter in which case, the exchanged power from the primary grid still occupies the main percentage. This indicates the developing potential for the new energy generation. It is transparent that, in the good contaminationdiffusing weather conditions, the power from the primary 


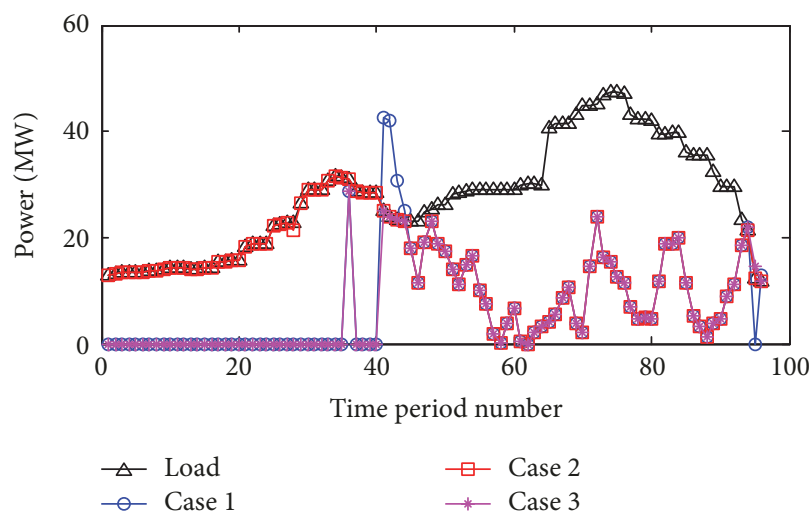

(a)

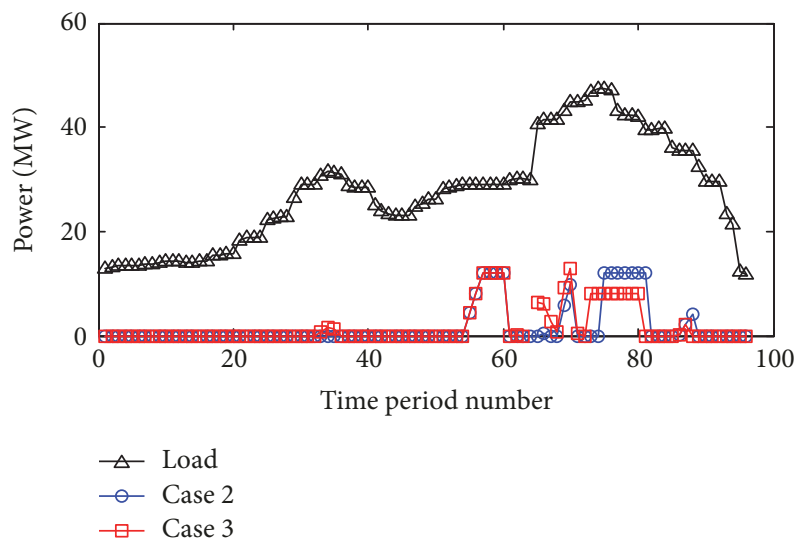

(c)

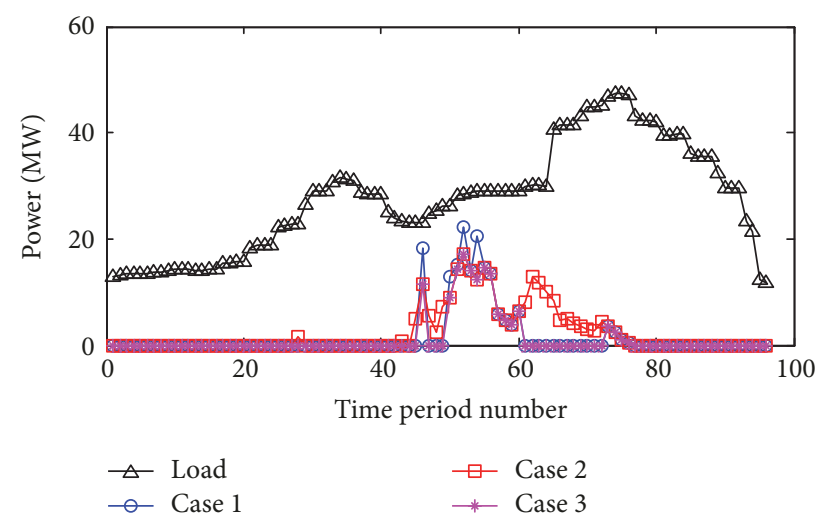

(b)

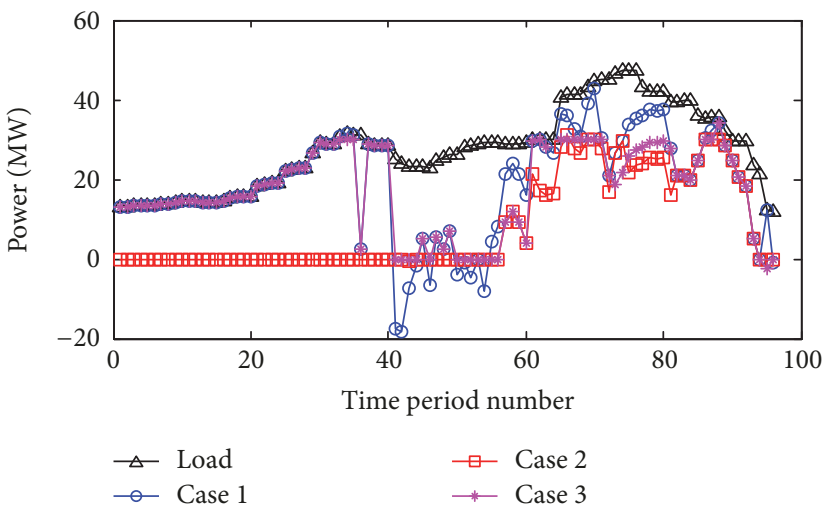

(d)

FIGURE 8: Power dispatching comparison, respectively, for (a) wind generation, (b) photovoltaic generation, (c) battery discharging, and (d) exchanged power from primary grid.

grid is the most, while in the smog weather condition it will be the least due to the utmost output of new energy generation and battery discharging.

It is also suggested by the cost comparison among the three cases that the normal power dispatching will spend a medium cost. It is more expensive than the good contamination-diffusing weather condition while it is relatively more cost-efficient than the smog weather conditions. The general cost in normal condition is $13.3 \%$ more expensive than the good contamination-diffusing weather condition, while it is $11.5 \%$ cheaper than the smog weather condition.

This can be thought as reasonable on the economic and the environmental sides. During the smog weather period, people are suffering the air pollution and the government is supposed to reduce the emission of all kinds of contamination. Consequently, the exchanged power from the primary grid generated by the thermal power plant based on fossil fuel consumption is supposed to be used as less as possible, while the new energy generation and the storage system are supposed to output their utmost capacity. It is easy to understand that in this case the cost will be higher due to the photovoltaic and wind generation performance which has a more expensive price than the traditional thermal power generation. On the contrary, under the good contaminationdiffusing weather conditions, the microgrid is able to use as much power from the primary grid as possible to reduce the cost, as long as it meets the environmental management request. Intermediately, the normal condition takes a balanced performance for the power dispatching to boost not only an economic effect but also a qualified environment protecting factor at the same time.

Under the guidance of the policy which has strong Chinese national condition features, contaminant emission has been greatly reduced due to the power dispatching optimization in smog weather condition as well as other measurements. Figure 9(a) shows the contaminant density developing trend in Suzhou Industrial Part, while Figures 9(b)-9(d) represent the general graphical change of Suzhou Industrial Part in the recent three years. Though the air condition improvement relies on many factors, it still shows the effectiveness of the proposed power dispatching method because many other measures such as using the electrical heating system in winter instead of coal combustion still depend on the electric power output. Therefore, generally, the proposed method in this paper is qualified and can be transferred to other similar cases. 


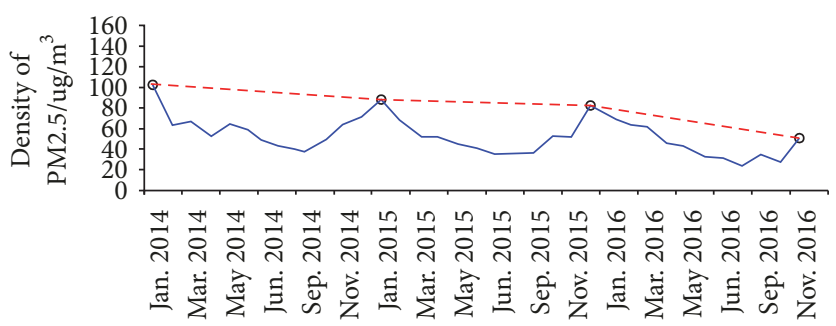

(a) Contaminant developing tendency

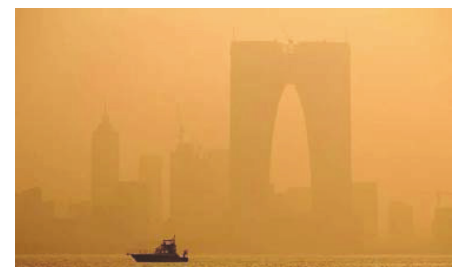

(b) 2015 winter

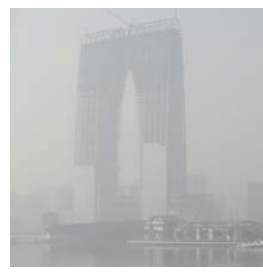

(c) 2016 winter

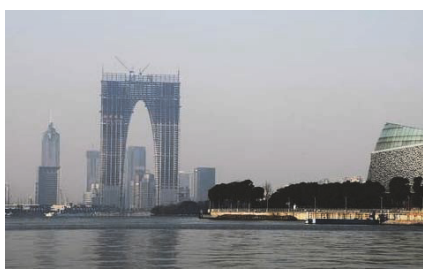

(d) 2017 winter

FIgURE 9: Comparison of the smog reduction around the Gate of the Orient.

\section{Conclusion}

This paper proposed a study on the optimized power dispatching in microgrids based on PSO algorithm. The whole work is on the basis of the weighted factor variation of the objective function due to different weather conditions. Three cases, that is, the good contamination-diffusing weather condition, the smog weather condition, and normal condition, are studied, respectively.

The case study on the Suzhou Industrial Part confirms the effectiveness of the proposed method. It is shown that, in the smog weather cases, the photovoltaic generation and the wind generation, as well as the storage system, will output their utmost power to reduce using the exchanged power from the primary grid for the sake of environment protection. In this case, however, the general cost will be the highest due to the power consumption of the new energy generation which has a more expensive price than the traditional coalbased thermal power generation. On the contrary, in the good contamination-diffusing weather cases, the strategy will be opposite. The exchanged power from the primary grid is intensively used to obtain the most economic-efficient effect. Consequently, the cost in this case is the lowest. Typically, in normal condition, the power dispatching is performed focusing on the balance between the economy and the environmental management, and the cost will be the medial one. The proposed method, as well as the case study, is qualified to be transferred to other similar cases.

\section{Conflicts of Interest}

The authors declare that there are no conflicts of interest regarding the publication of this paper.

\section{Acknowledgments}

This work is financed by State Grid Jiangsu Electric Power Company's scientific project (J2016015), the National Natural
Science Foundation of China (51307058), and Chinese Fundamental Research Funds for the Central Universities (2017MS160).

\section{References}

[1] A. Elrayyah, Y. Sozer, and M. E. Elbuluk, "Modeling and control design of microgrid-connected PV-based sources," IEEE Journal of Emerging and Selected Topics in Power Electronics, vol. 2, no. 4, pp. 907-919, 2014.

[2] G. Ji, W. Wu, and B. Zhang, "Robust generation maintenance scheduling considering wind power and forced outages," IET Renewable Power Generation, vol. 10, no. 5, pp. 634-641, 2016.

[3] N. Nikmehr and S. Najafi-Ravadanegh, "Optimal operation of distributed generations in micro-grids under uncertainties in load and renewable power generation using heuristic algorithm," IET Renewable Power Generation, vol. 9, no. 8, pp. 982990, 2015.

[4] J. Lin, C. Wan, and Y. Song, "Situation awareness of active distribution network: Roadmap, technologies, and bottlenecks," CSEE Journal of Power and Energy Systems, vol. 2, no. 3, pp. 3542, 2016.

[5] M. Nick, R. Cherkaoui, and M. Paolone, "Optimal allocation of dispersed energy storage systems in active distribution networks for energy balance and grid support," IEEE Transactions on Power Systems, vol. 29, no. 5, pp. 2300-2310, 2014.

[6] A. Ortega and F. Milano, "Generalized model of vsc-based energy storage systems for transient stability analysis," IEEE Transactions on Power Systems, vol. 31, no. 5, pp. 3369-3380, 2016.

[7] D. Debnath and K. Chatterjee, "Two-Stage Solar PhotovoltaicBased Stand-Alone Scheme Having Battery as Energy Storage Element for Rural Deployment," IEEE Transactions on Industrial Electronics, vol. 62, no. 7, pp. 4148-4157, 2015.

[8] J. M. Guerrero, M. Chandorkar, T.-L. Lee, and P. C. Loh, "Advanced control architectures for intelligent microgridspart I: decentralized and hierarchical control," IEEE Transactions on Industrial Electronics, vol. 60, no. 4, pp. 1254-1262, 2013. 
[9] J. M. Guerrero, P. C. Loh, T.-L. Lee, and M. Chandorkar, "Advanced control architectures for intelligent microgridsPart II: Power quality, energy storage, and AC/DC microgrids," IEEE Transactions on Industrial Electronics, vol. 60, no. 4, pp. 12631270, 2013.

[10] S. Maharjan, Q. Zhu, Y. Zhang, S. Gjessing, and T. Başar, "Demand response management in the smart grid in a large population regime," IEEE Transactions on Smart Grid, vol. 7, no. 1, pp. 189-199, 2016.

[11] N. Ghaffarzadeh and S. Bijani, "Dual surface sliding mode controller for photovoltaic systems enhanced by a Ripple domain search maximum power point tracking algorithm for fast changing environmental conditions," IET Renewable Power Generation, vol. 10, no. 5, pp. 611-622, 2016.

[12] C. Wei, Z. M. Fadlullah, N. Kato, and A. Takeuchi, "GT-CFS: A game theoretic coalition formulation strategy for reducing power loss in micro grids," IEEE Transactions on Parallel and Distributed Systems, vol. 25, no. 9, pp. 2307-2317, 2014.

[13] A. Mukherjee and V. Mukherjee, "Solution of optimal reactive power dispatch by chaotic krill herd algorithm," IET Generation, Transmission \& Distribution, vol. 9, no. 15, pp. 2351-2362, 2015.

[14] C. Battistelli, L. Baringo, and A. J. Conejo, "Optimal energy management of small electric energy systems including V2G facilities and renewable energy sources," Electric Power Systems Research, vol. 92, pp. 50-59, 2012.

[15] Q. Jiang, M. Xue, and G. Geng, "Energy management of microgrid in grid-connected and stand-alone modes," IEEE Transactions on Power Systems, vol. 28, no. 3, pp. 3380-3389, 2013.

[16] M. Mao, P. Jin, N. D. Hatziargyriou, and L. Chang, "Multiagentbased hybrid energy management system for microgrids," IEEE Transactions on Sustainable Energy, vol. 5, no. 3, pp. 938-946, 2014.

[17] D. E. Olivares, C. A. Canizares, and M. Kazerani, "A centralized energy management system for isolated microgrids," IEEE Transactions on Smart Grid, vol. 5, no. 4, pp. 1864-1875, 2014.

[18] M. Marzband, N. Parhizi, M. Savaghebi, and J. M. Guerrero, "Distributed Smart Decision-Making for a Multimicrogrid System Based on a Hierarchical Interactive Architecture," IEEE Transactions on Energy Conversion, vol. 31, no. 2, pp. 637-648, 2016.

[19] X. Hu and T. Liu, "Co-optimisation for distribution networks with multi-microgrids based on a two-stage optimisation model with dynamic electricity pricing," IET Generation, Transmission \& Distribution, vol. 11, no. 9, pp. 2251-2259, 2017.

[20] C. Battistelli, Y. P. Agalgaonkar, and B. C. Pal, "Probabilistic Dispatch of Remote Hybrid Microgrids Including Battery Storage and Load Management," IEEE Transactions on Smart Grid, vol. 8, no. 3, pp. 1305-1317, 2017.

[21] N. Nikmehr and S. Najafi Ravadanegh, "Optimal Power Dispatch of Multi-Microgrids at Future Smart Distribution Grids," IEEE Transactions on Smart Grid, vol. 6, no. 4, pp. 1648-1657, 2015.

[22] G. Liao, "Solve environmental economic dispatch of smart microgrid containing distributed generation system-Using chaotic quantum genetic algorithm," International Journal of Electrical Power \& Energy Systems, vol. 43, no. 1, pp. 779-787, 2012.

[23] M. Koller, T. Borsche, A. Ulbig, and G. Andersson, "Defining a degradation cost function for optimal control of a battery energy storage system," in Proceedings of the 2013 IEEE Grenoble Conference PowerTech, POWERTECH 2013, Grenoble, France, June 2013.
[24] W. Su, J. Wang, and J. Roh, "Stochastic energy scheduling in microgrids with intermittent renewable energy resources," IEEE Transactions on Smart Grid, vol. 5, no. 4, pp. 1876-1883, 2013.

[25] M. Varadarajan and K. S. Swarup, "Solving multi-objective optimal power flow using differential evolution," IET Generation, Transmission \& Distribution, vol. 2, no. 5, pp. 720-730, 2008.

[26] X. Zhang, J. Sun, Y. Wang, and etal., "Factors contributing to haze and fog in China," Chinese Science Bulletin (Chinese Version), vol. 58, no. 13, p. 1178, 2013.

[27] S. L. Zhang, Y. H. Wang, Y. Li, and P. F. Zhang, "Spatial distribution of haze pollution and its influencing factors," China Population, Resources and Environment, vol. 27, no. 9, pp. 15-22, 2017 (Chinese).

[28] Z. Tian, Z. Yang, and R. Cai, "Study on contribution of power industry for regional energy conservation and carbon intensity reduction goal," Electric Power, vol. 48, no. 3, pp.150-155, March 2015 (Chinese).

[29] S. Zhang and Q. Tian, "Sensitivity Analysis of Micro-grid Scheduling to on-Grid Price and DG Subsidies," Electric Power, vol. 49, no. 4, pp. 174-180, April 2016 (Chinese).

[30] National Development and Reform Commission, Environmental protection department, and National energy bureau, Energy conservation and emission reduction and upgrading plan, Chinese National Development and Reform Commission policy, http://bgt.ndrc.gov.cn/zcfb/201409/t20140919_626242.html, Sep., 2014.

[31] S. García-Galán, R. P. Prado, and J. E. Muñoz Expósito, "Rules discovery in fuzzy classifier systems with PSO for scheduling in grid computational infrastructures," Applied Soft Computing, vol. 29, pp. 424-435, 2015. 


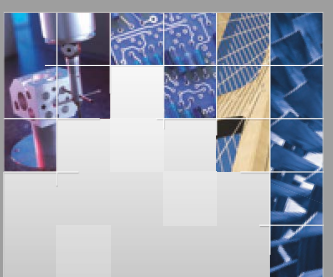

\section{Enfincering}
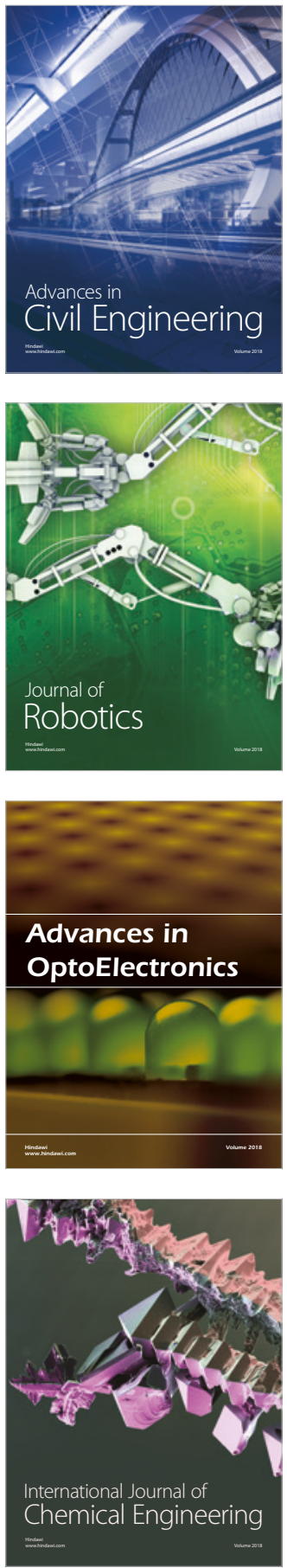

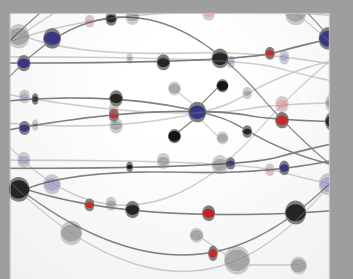

\section{Rotating \\ Machinery}

The Scientific World Journal

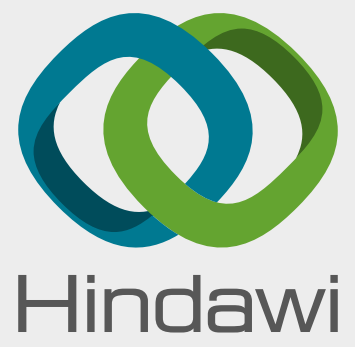

Submit your manuscripts at

www.hindawi.com
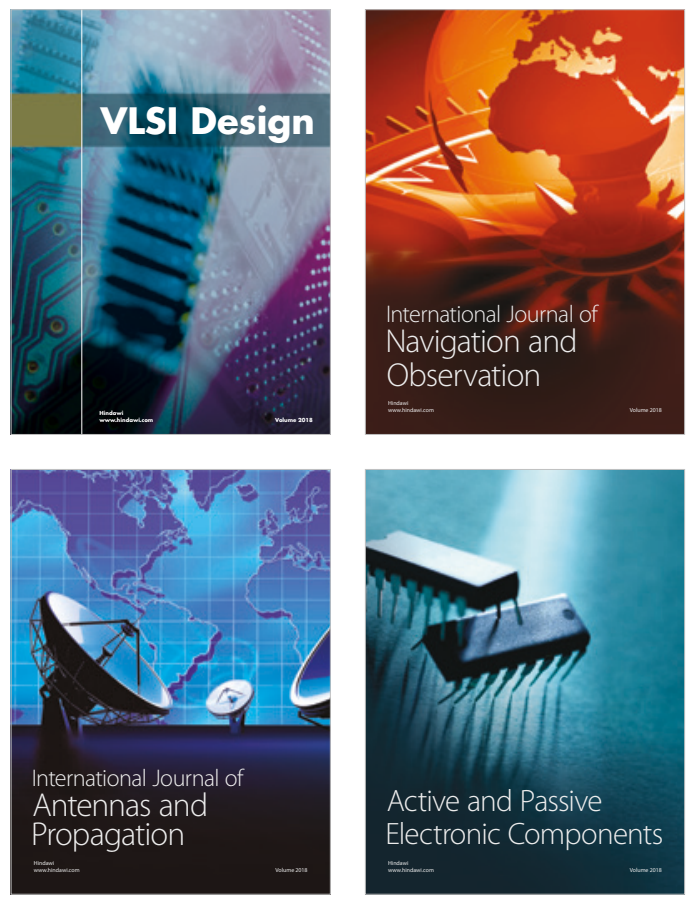
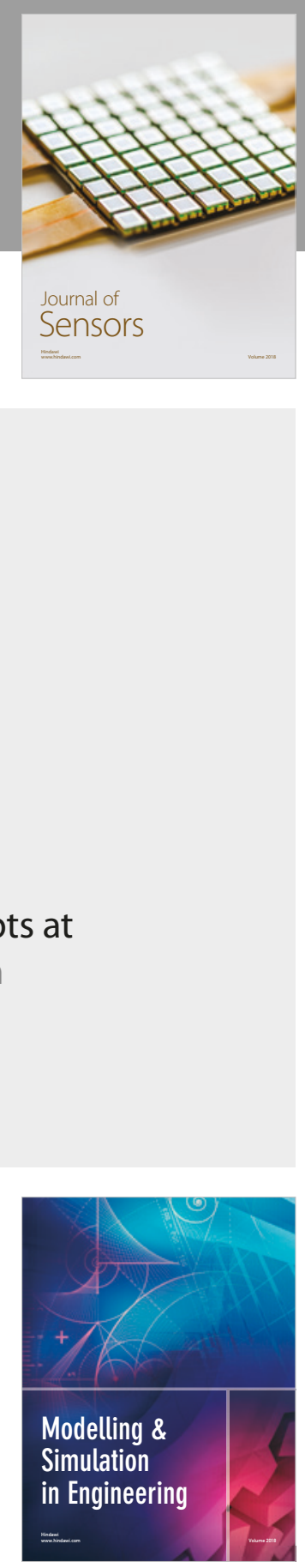

\section{Advances \\ Multimedia}
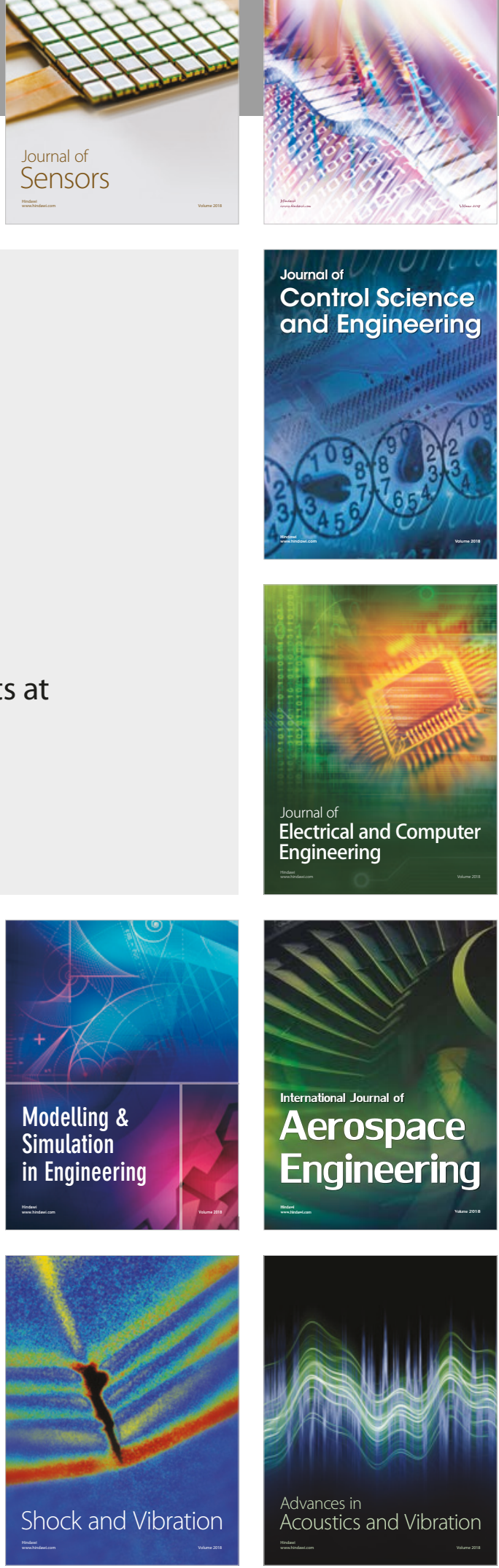